\title{
ADME Evaluation in Drug Discovery. 6. Can Oral Bioavailability in Humans Be Effectively Predicted by Simple Molecular Property-Based Rules?
}

\author{
Tingjun Hou, ${ }^{*}$, Junmei Wang, ${ }^{\ddagger}$ Wei Zhang, ${ }^{\S}$ and Xiaojie $\mathrm{Xu}^{\ddagger}$ \\ Department of Chemistry and Biochemistry, Center for Theoretical Biological Physics, University of \\ California at San Diego, La Jolla, California 92093, College of Chemistry and Molecular Engineering, \\ Peking University, Beijing 100871, P. R. China, and Department of Molecular Biology, \\ The Scripps Research Institute, La Jolla, California 92037
}

Received August 14, 2006

\begin{abstract}
A critically evaluated database of human oral bioavailability for 768 chemical compounds is described in this study (http://modem.ucsd.edu/adme), which provides the scientific community a publicly available and reliable source for developing predictive models of human oral bioavailability. The correlations between several important molecular properties and human oral bioavailability were investigated and compared with an earlier report by analyzing the rat oral bioavailability data (J. Med. Chem. 2002, 45, 2615). We showed that the percentages of compounds meeting the criteria based on molecular properties does not distinguish compounds with poor oral bioavailability from those with acceptable values, which may suggest that no simple rule based on molecular properties can be used as general filters to predict oral bioavailability with high confidence. A data set of intestinal absorption was also examined and compared with that of oral bioavailability. The performance of these rules based on molecular properties in the prediction of intestinal absorption is obviously much better than that of oral bioavailability in term of false positive rate, and, therefore, the applications of the "rule-based" approaches on the prediction of human bioavailability should be very cautious.
\end{abstract}

\section{INTRODUCTION}

In the development of drugs intended for oral use, good drug absorption and appropriate drug delivery are very important. ${ }^{1}$ About $30 \%$ of oral drugs fail in development due to poor pharmacokinetics. ${ }^{2}$ Among the pharmacokinetic properties, a low and highly variable bioavailability is indeed the main reason for stopping further development of the drug. Oral bioavailability of a drug is related to many factors, such as dissolution in the gastrointestinal tract, intestinal membrane permeation, and intestinal/hepatic first-pass metabolism.

The application of combinatorial synthesis and highthroughput screening technologies in the pharmaceutical industry has resulted in the production of a very large number of pharmacological active molecules that require pharmacokinetic assessment. Thus, predictions of bioavailability and bioavailability-related properties, such as intestinal absorption, solubility, the effect of transporter proteins, metabolism based on cytochrome P450 proteins, etc., are areas in need of progress to aid pharmaceutical drug development. An in silico model for predicting oral bioavailability is very important, both in the early stage of drug discovery to select the most promising compounds for further optimization and in the later stage to identify candidates for further clinical development.

The in silico prediction of oral bioavailability may be pioneered by the Lipinski's 'Rule-of-Five': if two out of

\footnotetext{
* Corresponding author e-mail: tingjunhou@hotmail.com.

$\dagger$ University of California at San Diego.

$\doteqdot$ Peking University.

$\S$ The Scripps Research Institute.
}

the following five parameters are out of the range, then a poor absorption or permeability is possible: the molecular weight is over 500, hydrogen bond donors are more than 5, the calculated octanol-water partition coefficient is over 5 (CLOGP) or 4.15 (MLOGP), and hydrogen bond acceptors are more than 10 . The 'Rule-of-Five' should be considered as a qualitative predictor of absorption and permeability. ${ }^{3}$ In recent years, several prediction models of oral bioavailability based on quantitative-structure activity relationship (QSAR) analysis have been reported. ${ }^{4-8}$ In 2000, Andrews and co-workers reported a regression model to predict bioavailability. ${ }^{5}$ Compared to Lipinski's Rule-of-Five, the false negative predictions were reduced from $5 \%$ to $3 \%$, while the false positive predictions decreased from $78 \%$ to $53 \%$. But it should be noted that in Andrew's regression model, 85 descriptors were used, and thus overfitting problem may exist. In 2000, Yoshida et al. proposed a classification model for bioavailability based on three descriptors related to distribution coefficient and 15 structural descriptors. This classification model can give a correct rate of $60 \%$ for the tested compounds. The authors believed that 15 structural descriptors used in the model were closely related to wellknown metabolic processes. In 2002 Veber et al. reported the findings from a study of rat availability data, acquired by GlaxoSmithKline, for 1100 drug candidates. ${ }^{6}$ They found that molecules possessing fewer than 10 rotatable bonds and having a polar surface area less than $140 \AA^{2}$ (or H-bond count less than 12) generally showed oral bioavailability in rats exceeding 20\%. In 2004, Lu and co-workers checked the relationship of rotatable bond count and polar surface area (PSA) with the oral bioavailability in rats for 434 compounds 
and compared it with Veber's work. ${ }^{8}$ Lu found that the correlations were dependent on the calculation methods. Recently, Martin proposed a score to predict bioavailability based on several molecular properties, including polar surface area (PSA), Rule of Five, and molecular charged state. ${ }^{4}$ The authors believed that the bioavailability score might be used in very early drug discovery for selecting compounds to purchase or synthesize. Generally, Veber's work as well as Lu's work and Martin's work supports the same conclusion: bioavailability may be well predicted by simple molecular properties.

The rules proposed by Veber et al. ${ }^{6}$ as well as the two following models proposed by $\mathrm{Lu}$ et al. ${ }^{8}$ and Martin et al. ${ }^{4}$ were based on rat data and that their applications to dosing in human may by limited by the lack of correlation of absorption between species. ${ }^{9}$ It was of great interest to evaluate whether Veber's rules could be applied to the prediction of human oral bioavailability. Therefore, 768 compounds, with oral bioavailability data, were collected into a database, and the relationships between the human bioavailability and several calculated molecular properties were explored. This set of data has been collected from the published literature and is the largest one of its kind available to the public. It allows the scientific community to develop and test new prediction rules or models. Our analysis suggested that there are no simple rules based on molecular properties that can be used to predict bioavailability reliably.

\section{METHODS}

1. Database of Oral Bioavailability in Human. The oral bioavailability data in human for 768 compounds were collected from 185 published papers. The large size of the database ensures the statistical significance of our analysis. The structurally heterogeneous molecules in the database cover a wide range of molecular size and lipophilicity. The structures of the compounds were built using the Cerius2 molecular simulation package. Each molecule in the database was optimized by using molecular mechanics with the MMFF force field, ${ }^{10}$ and the termination condition was the rms of potential energy gradient smaller than $0.01 \mathrm{kcal} \cdot \AA^{-1} \cdot \mathrm{mol}^{-1}$. All molecules were then saved to a MACCS sdf file and a SMILES database for further analysis.

The 2-D structures, oral bioavailability data, and the corresponding references for all compounds can be downloaded from http://modem.ucsd.edu/adme. For compounds with different reported experimental bioavailability values, the average values were used to reduce bias of different experiments.

2. Molecular Properties. There are totally eight molecular properties used in this analysis, which include molecular weight (MW), topological polar surface area (TPSA), rotatable bond count $\left(N_{\text {rot }}\right)$, H-bond donor count $\left(N_{\mathrm{HBD}}\right), \mathrm{H}$-bond acceptor count $\left(N_{\mathrm{HBA}}\right)$, total H-bond count $\left(N_{\mathrm{HB}}\right)$, octanolwater partitioning coefficient $(\log P)$, and apparent partition coefficient $(\log D)$ at $\mathrm{pH}=7.4$.

TPSA was calculated using the parameters originally proposed by Ertl et al., ${ }^{11}$ which were developed to calculate polar surface area of a molecule based on its 2-D molecular bonding information. $N_{\text {rot }}$ is the number of bonds in a molecule (all terminal $\mathrm{H}$ atoms are ignored) allowed to rotate in molecular mechanics. $N_{\text {rot }}$ was computed using Cerius2.
TPSA, $N_{\mathrm{HBD}}$, and $N_{\mathrm{HBA}}, \log P$, and $\log D$ at $\mathrm{pH}=7.4$ were calculated using ACD/labs (version 9.0). The molecular names and the analyzed properties are listed in Table S1. The distributions of the bioavailability data and the studied properties are shown in Figure S1 in the Supporting Information.

\section{RESULTS AND DISCUSSION}

The distributions of the bioavailability values and the calculated properties are shown in Figure S1 in the Supporting Information. In Veber's work, the authors found that the effect of molecular rigidity, represented by rotatable bond numbers, on oral bioavailability is independent of molecular weight. ${ }^{6}$ Confirming Veber, we also observed an obvious correlation between the rotatable bond number and the molecular weight $(r=0.65)$. To explore the influence of molecular properties on bioavailability, we divided our data set into bioavailability quartiles following Veber's protocol. ${ }^{6}$ The resulting quartile comparisons are shown in Table $\mathrm{S} 1$ in the Supporting Information. As shown in Figure S1 and Table S1, the average oral bioavailablity of human $(51 \%)$ obviously preferred much larger values than that of rats (29\%). ${ }^{6}$ The average molecular weight is 347 (human) vs 480 (rat), the average number of rotatable bonds is $6.4 \mathrm{vs}$ 8.4, the average topological polar surface area is $89 \AA^{2}$ vs $102 \AA,{ }^{2}$ and the average H-bond total count is 7.6 vs 9.2, suggesting that there is a large difference in the structural characteristics of the two data sets. Overall, the human data set includes more compounds with lower molecular weight and higher bioavailability than the rat data set.

In Table S1, the analysis for three subsets based on different molecular weight regions is also included. The quartile averages confirm that higher oral bioavailability is indeed associated with lower MW, but the analysis also reveals that higher oral bioavailability is also associated with lower $N_{\text {rot }}$, lower $N_{\mathrm{HB}}$, and lower TPSA. In fact, MW is obviously correlated with TPSA, $N_{\text {rot }}$, and $N_{\mathrm{HB}}$. As shown in Table S1, no single molecular property was observed to have effective correlation with oral bioavailability.

In Veber's work, ${ }^{6}$ two simple rules were proposed to identify compounds with excepted oral bioavailability: If a rat oral bioavailability of $\geq 20-40 \%$ is considered acceptable, then it appears that TPSA $\leq 140 \AA^{2}$ (or sum of H-bond donors and acceptors $\leq 12$ ) combined with $N_{\text {rot }} \leq 10$ is an efficient and selective criterion. It will be very interesting to check the performance of these two rules on the human data. Similar rules were proposed by $\mathrm{Lu}$ and co-workers based on two different methods to compute $N_{\text {rot }}$ and PSA. The rules proposed by Veber et al. and Lu et al. are quite similar, ${ }^{6,8}$ only the threshold values for PSA and $N_{\text {rot }}$ are different due to the usage of different methods and the dependence on the different data set. Here, the rules proposed by Veber were validated because both Veber and us applied the same definition for TPSA. The success of the rules applied to the human data was evaluated by plotting the cumulative fraction meeting the rule as a function of increasing oral bioavailability (Figure 1). In the human data set, $84 \%$ of compounds with bioavailability exceeding $20 \%$ meet the criteria proposed by Veber et al. (TPSA $\leq 140 \AA^{2}$ and $N_{\text {rot }} \leq 10$ or $n_{\mathrm{HB}} \leq 12$ and $N_{\text {rot }} \leq 10$ ). By comparison, Veber found $80 \%$ of their compounds meeting these criteria. 


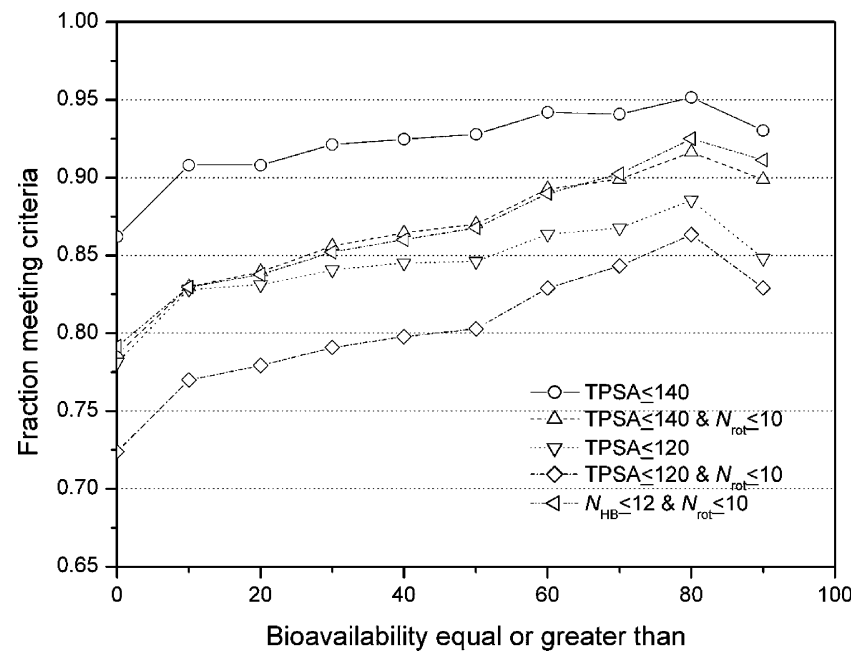

Figure 1. The performance of various rules for selecting orally bioavailable compounds.

The cumulative fractions using the other three rules (TPSA $\leq 140 \AA,{ }^{2}$ TPSA $\leq 120 \AA,{ }^{2}$ TPSA $\leq 120 \AA^{2}$ and $N_{\text {rot }} \leq 10$ ) are also shown in Figure 1. When we used a more flexible rule (TPSA $\leq 140 \AA^{2}$ ), more compounds with bioavailability larger than $20 \%$ were selected, but more compounds with bioavailability smaller than $20 \%$ were also identified. When we used a more stringent rule (TPSA $\leq 120 \AA^{2}$ and $N_{\text {rot }} \leq$ $10)$, the percentage of the selected compounds decreases obviously.

In Figure 1, it can be seen that Veber's rules have good coverage for compounds with bioavailability larger than $20 \%$, but we cannot draw the conclusion if these rules are really effective to predict the bioavailability because a good model requires the prediction with a good balance between a false positive rate and a false negative rate. Therefore, we validated the performance of these rules on the favorably bioavailable group (bioavailability equal or larger than 20\%) and the unfavorably bioavailable group (bioavailability smaller than $20 \%$ ), respectively. The values of each property were divided into three different regions, and the percentage of compounds meeting the property distribution is shown in Figure S2 in the Supporting Information. For each single property, in the relatively lower region, such as $\mathrm{MW} \leq 400, N_{\text {rot }} \leq 7$ or TPSA $\leq 120 \AA,^{2}$ the percentage of the selected compounds with bioavailability $\geq 20 \%$ is higher than those with bioavailability $<20 \%$. While in the relatively larger regions, the results are usually adverse. If we used each single property as the rule to identify favorably bioavailable compounds, the correct rate is high (larger than 80\%). Meanwhile, the false positive rate is also very high. If we included two properties, $N_{\text {rot }}$ and TPSA, in the rule, the false positive rate can be reduced (see Figure S2). The false positive rate using the combined rule of $N_{\text {rot }} \leq 10$ and TPSA $\leq 140 \AA^{2}$ is about $60 \%$. That is to say, about 100 compounds with bioavailability $<20 \%$ cannot be correctly classified. Among these 170 compounds with bioavailability smaller than $20 \%, 112$ compounds meet the Lipinski's 'Rule of Five'. Among these 112 compounds, 85 cannot be correctly predicted by Veber's rule. According to the above analysis, we found that no rules based on molecular properties can effectively identify these unfavorably bioavailable compounds.
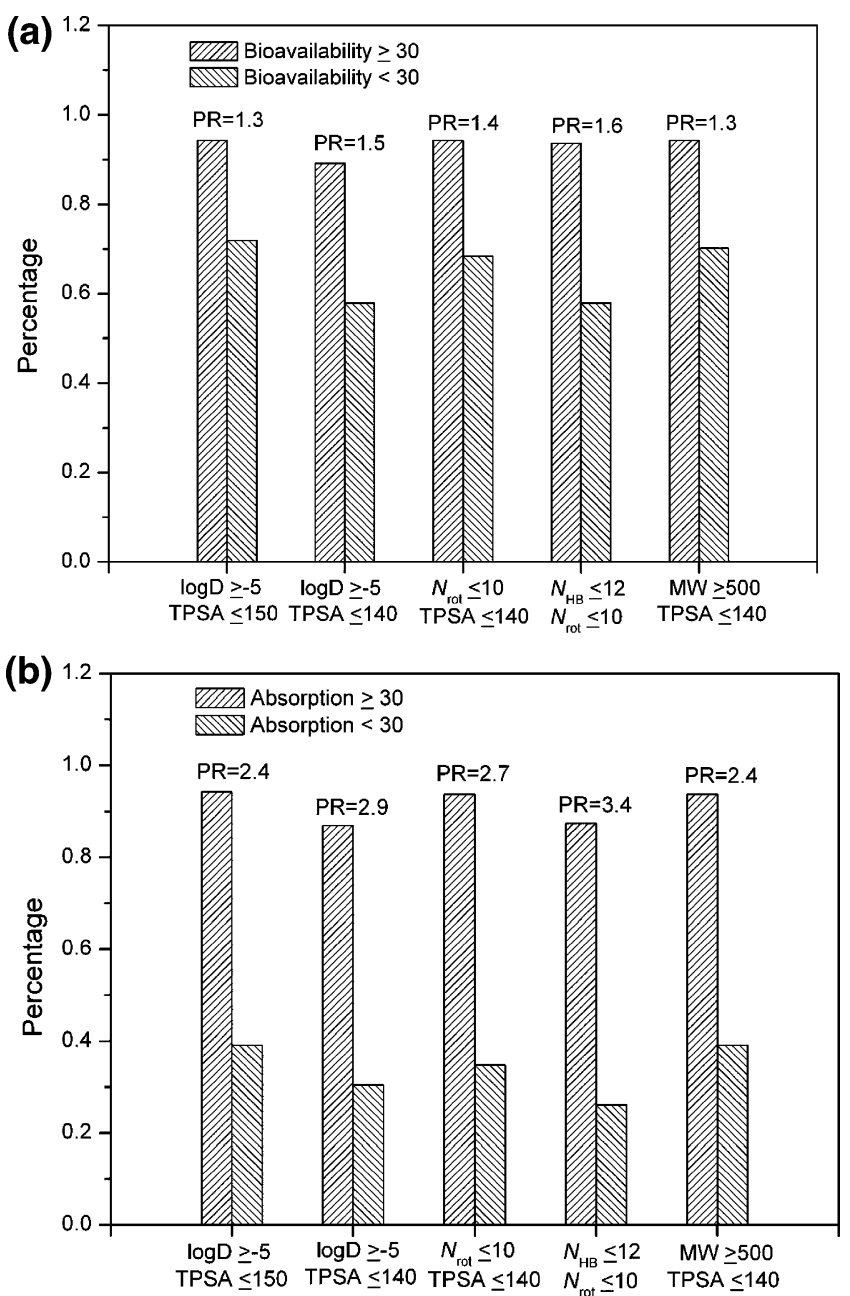

Figure 2. The percentage of compounds for compounds with (a) oral bioavailability $\geq 30 \%$ or $<30 \%$ and (b) oral absorption $\geq 30 \%$ or $<30 \%$ meeting the defined rules.

Figure S3 in the Supporting Information shows the scatter plot of bioavailability $(\% \mathrm{~F})$ vs intestinal absorption $(\% \mathrm{HIA})^{12-14}$ for 214 compounds. The \%HIA values for these 214 compounds are listed in Table S3 in the Supporting Information. In Figure S3 it can be observed that many compounds are located far from the diagonal line. These are most likely that these compounds have significant hepatic first-pass metabolism effect. The correlations between the intestinal absorption and the studied properties (Figure S4 in the Supporting Information) indicate that intestinal absorption is closely negatively correlated with $N_{\mathrm{HBD}}(r=-0.63), N_{\mathrm{HBA}}$ $(r=-0.61), N_{\mathrm{HB}}(r=-0.66)$, and TPSA $(r=-0.65)$. In fact, all these four descriptors are high correlated. In the prediction of membrane permeability, PSA or H-bond-related properties are usually the most important descriptors. ${ }^{15}$ Drug lipophilicity is widely used as a predictor of membrane permeability since it is assumed that drug partitioning into the (lipophilic) cell membranes is a rate-determining process for passive membrane permeation. Our observations also proved that $\log D$ is also positively correlated with intestinal absorption $(r=0.51)$. Both of MW and $N_{\text {rot }}$ show negatively correlation with intestinal absorption, but their correlation coefficients are very low $(r=-0.37$ and -0.23$)$. For MW, 500 , a currently popular delimiter, shows some effectiveness to distinguish some low absorbed compounds (HIA\% < $20 \%$ ) from the higher absorbed ones. Here, we compared 
the performance of some property-based rules to predict intestinal absorption and bioavailability. We divided the 214 compounds into two subgroups: a favorable group (intestinal absorption or bioavailability is equal or larger than 30\%) and an unfavorable group (intestinal absorption or bioavailability is lower than $30 \%$ ). In total, we used five different rules based on TPSA, MW, $N_{\text {rot }}$, and $\log D$. The percentage of compounds in the favorable group and the unfavorable group selected by these rules are shown in Figure 2. The percentage of the correct predictions for compounds in the favorable group of bioavailability is about $89-94 \%$. This percentage is consistent with the results reported by Veber and co-workers. ${ }^{6}$ But for the 57 compounds in the unfavorable group, the percentage of the correct predictions is very low, which is indicated by the high false positive rate (58$72 \%$ ). Compared with the prediction of bioavailability, the prediction of intestinal absorption using these rules is much better. The percentage of the correct predictions for high bioavailability, across the five rules, varied between $89 \%$ and $94 \%$, which is comparative to that for compounds in the favorable group of absorption. But compared with the prediction of intestinal absorption $(26-39 \%)$, the prediction of bioavailability on the unfavorable group is much lower. Here, we defined a parameter:prediction ratio (PR), which is the ratio of the compounds meeting the rules in the favorable group and those meeting the rules in the unfavorable group. The PR value of the prediction of intestinal absorption (2.4-3.4) is significantly better than that of the prediction of bioavailability (1.3-1.6). In these five rules, the rule ' $N_{\mathrm{HB}} \leq 12$ and $N_{\text {rot }} \leq 10$ ' performs best for both of the prediction of bioavailability and that of intestinal absorption. The prediction of intestinal absorption using the rule ' $N_{\mathrm{HB}} \leq 12$ and $N_{\text {rot }}$ ' shows that 6 candidates in compounds with HIA\% of $<30 \%$ and 24 candidates in compounds with HIA $\%$ of $\geq 30 \%$ are misclassified. Careful analysis of these misclassified compounds shows that absorption of 4 misclassified compounds with HIA\% $<30 \%$ and 11 compounds with HIA $\% \geq 30 \%$ are actively transported, dose-limited, or dose-dependent. ${ }^{13}$ For example, for 4 misclassified compounds with HIA\% $<30 \%$, foscarnet is carrier-mediated transported, and olsalazin, chlorothiazide, and acyclovir are dose-limited or dose-dependently absorbed. If we eliminate these misclassified molecules from the data set, then the prediction of intestinal absorption should be improved further. So the rules based on molecular properties can be used as effective filters for the estimation of oral absorption, especially intestinal absorption of passive diffusion, but not for oral bioavailability. The reason is that the hepatic metabolism cannot be effectively considered by these molecular properties, and thus highly metabolized compounds may not be well predicted by these simple property-based rules.

Note: The human oral availability database can be downloaded from the Web site http://modem.ucsd.edu/adme. The database is password protected, and the password to uncompress the database is bioteam773.

\section{ACKNOWLEDGMENT}

T.H. is supported by a CTBP postdoctoral scholarship. We thank Prof. J. Andrew McCammon for providing access to Cerius2 molecular simulation package.
Supporting Information Available: Distribution of the molecular properties (Figure S1), percentage of compounds with an oral bioavailability of $20 \%$ or greater and those with an oral bioavailability smaller than $20 \%$ as a function of molecular properties (Figure S2), scatter plot of intestinal absorption vs human oral bioavailability (Figure S3), relationships between intestinal absorption and molecular properties (Figure S4), oral bioavailability quartile property average for the human oral bioavailability data set and for subsets divided on the basis of molecular weight (Table S1), calculated molecular properties for 768 compounds (Table S2), and \%HIA and $\% \mathrm{~F}$ values for 214 compounds (Table S3). This material is available free of charge via the Internet at http:// pubs.acs.org.

Note Added after ASAP Publication. This article was released ASAP on January 6, 2007 with minor errors in the Results and Discussion section. The correct version was posted on January 31, 2007.

\section{REFERENCES AND NOTES}

(1) Hou, T. J.; Xu, X. J. Recent development and application of virtual screening in drug discovery: An overview. Curr. Pharm. Des. 2004, $10,1011-1033$

(2) van de Waterbeemd, H.; Gifford, E. ADMET in silico modelling: Towards prediction paradise? Nat. Rev. Drug Discovery 2003, 2, 192204.

(3) Lipinski, C. A.; Lombardo, F.; Dominy, B. W.; Feeney, P. J. Experimental and computational approaches to estimate solubility and permeability in drug discovery and development settings. Adv. Drug Delivery Rev. 1997, 23, 3-25.

(4) Martin, Y. C. A bioavailability score. J. Med. Chem. 2005, 48, 31643170.

(5) Andrews, C. W.; Bennett, L.; Yu, L. X. Predicting human oral bioavailability of a compound: Development of a novel quantitative structure-bioavailability relationship. Pharm. Res. 2000, 17, 639-644.

(6) Veber, D. F.; Johnson, S. R.; Cheng, H. Y.; Smith, B. R.; Ward, K. W.; Kopple, K. D. Molecular properties that influence the oral bioavailability of drug candidates. J. Med. Chem. 2002, 45, 26152623.

(7) Yoshida, F.; Topliss, J. G. QSAR model for drug human oral bioavailability. J. Med. Chem. 2000, 43, 2575-2585.

(8) Lu, J. J.; Crimin, K.; Goodwin, J. T.; Crivori, P.; Orrenius, C.; Xing, L.; Tandler, P. J.; Vidmar, T. J.; Amore, B. M.; Wilson, A. G. E.; Stouten, P. F. W.; Burton, P. S. Influence of molecular flexibility and polar surface area metrics on oral bioavailability in the rat. J. Med. Chem. 2004, 47, 6104-6107.

(9) Sietsema, W. K. The Absolute Oral Bioavailability of Selected Drugs. Int. J. Clin. Pharmacol. Ther. 1989, 27, 179-211.

(10) Halgren, T. A. MMFF VI. MMFF94s option for energy minimization studies. J. Comput. Chem. 1999, 20, 720-729.

(11) Ertl, P.; Rohde, B.; Selzer, P. Fast calculation of molecular polar surface area as a sum of fragment-based contributions and its application to the prediction of drug transport properties. J. Med. Chem. 2000, 43, 3714-3717.

(12) Niwa, T. Using general regression and probabilistic neural networks to predict human intestinal absorption with topological descriptors derived from two-dimensional chemical structures. J. Chem. Inf. Comput. Sci. 2003, 43, 113-119.

(13) Deretcy, E.; Feher, M.; Schmidt, J. M. Rapid prediction of human intestinal absorption. Quant. Struct.-Act. Relat. 2002, 21, 493-506.

(14) Wessel, M. D.; Jurs, P. C.; Tolan, J. W.; Muskal, S. M. Prediction of human intestinal absorption of drug compounds from molecular structure. J. Chem. Inf. Comput. Sci. 1998, 38, 726-735.

(15) Hou, T. J.; Wang, J. M.; Zhang, W.; Wang, W.; Xu, X. Recent advances in computational prediction of drug absorption and permeability in drug discovery. Curr. Med. Chem. 2006, 13, 2653-2667. 